\title{
Estrategias de intervención de los factores de riesgo psicosocial de origen laboral: una visión desde terapia ocupacional
}

\author{
Estratégias de intervençâo do fator de risco psicossocial relacionadas ao \\ trabalho: uma visão da terapia ocupacional \\ Work-related psychosocial risk factor intervention strategies: \\ an occupational therapy view \\ Karen Danniela Medina Murillo ${ }^{a}$ (D), Olga Beatriz Guzmán Suárez (D), Jaime Moreno-Chaparro ${ }^{a}$ \\ ${ }^{a}$ Universidad Nacional de Colombia, Bogotá D. C., Colombia.
}

Cómo citar: Murillo, K. D. M., Guzmán Suárez, O. B., \& Moreno-Chaparro, J. (2020). Estrategias de intervención de los factores de riesgo psicosocial de origen laboral: una visión desde terapia ocupacional. Cadernos Brasileiros de Terapia Ocupacional, 28(2), 436-451. https://doi.org/10.4322/2526-

8910.ctoAO1934

\begin{abstract}
$\underline{\text { Resumen }}$
Introducción: Los factores psicosociales son interacciones dinámicas entre diferentes dimensiones del ser humano, quehacer, ocupación, ambiente, entre otros. La alteración de esta dinámica produce riesgos que impactan en la salud y bienestar de los trabajadores. Objetivo: Identificar y proponer estrategias de intervención enfocadas en los factores psicosociales a nivel laboral desde terapia ocupacional. Método: Estudio de enfoque cualitativo, transversal y bajo paradigma histórico hermenéutico. Se realizaron dos fases enfocadas en intervenciones psicosociales de origen laboral desde terapia ocupacional. La primera fase de búsqueda documental y la segunda, una encuesta a terapeutas ocupacionales colombianos sobre su experiencia específica en intervención del riesgo psicosocial. Se realiza análisis por triangulación. Resultados: Se identifican siete documentos con influencia teórica, práctica e histórica relevantes para la investigación y análisis. De la segunda fase, se validan 11 respuestas de terapeutas ocupacionales colombianos los cuales describen dos preguntas de interés para esta investigación relacionadas a factores protectivos o preventivos en cuanto a riesgos psicosociales de origen laboral. Acerca de la segunda pregunta describen acciones, intervenciones o actividades que desde su experticia profesional han considerado efectivos para la formulación de una estrategia de intervención de factores psicosociales. Se realiza una triangulación analítica donde se proponen estrategias derivadas de la búsqueda documental, las respuestas de la encuesta y la información compilada. Se identifican adicionalmente categorías de promoción, prevención, teoría y sustentación.
\end{abstract}


Conclusión: Se proponen trece estrategias de intervención enmarcadas en el objetivo y su especificidad en promoción de la salud, prevención de accidentes y enfermedades laborales, rehabilitación laboral y favorecimiento de la población trabajadora.

Palabras-clave: Gestión de Riesgos, Salud Laboral, Promoción de la Salud, Impacto Psicosocial, Servicios de Salud Mental, Terapia Ocupacional.

\section{$\underline{\text { Resumo }}$}

Introdução: Os fatores psicossociais são interaçôes dinâmicas entre diferentes dimensões do ser humano, tarefa, ocupação, meio ambiente, entre outros. A alteração dessa dinâmica produz riscos que impactam a saúde e o bem-estar dos trabalhadores. Objetivo: Identificar e propor estratégias de intervenção voltadas para fatores psicossociais no nível ocupacional a partir da terapia ocupacional. Método: Estudo do paradigma histórico qualitativo, transversal e de baixa hermenêutica. Duas fases focalizaram intervenções psicossociais de origem ocupacional a partir da terapia ocupacional. A primeira fase foi busca documental e a segunda um levantamento dos terapeutas ocupacionais colombianos sobre sua experiência específica na intervenção de risco psicossocial. A análise foi realizada por triangulação dos dados. Resultados: Foram identificados sete documentos com influência teórica, prática e histórica para a investigação e análise. A partir da segunda fase, foram validadas 11 respostas de terapeutas ocupacionais colombianos, que descreveram duas questóes de interesse relacionadas a fatores protetores ou preventivos em relação aos riscos psicossociais de origem laboral. Descrevem açóes, intervenções ou atividades em que em sua perícia profissional consideraram eficazes para a formulação de uma estratégia de intervenção psicossocial. Uma triangulação analítica foi realizada entre as estratégias derivadas da pesquisa documental, as respostas da pesquisa e as informaçôes compiladas. As categorias de promoçáo, prevenção, teoria e apoio também foram identificadas. Conclusão: Treze estratégias de intervençáo sáo propostas enquadradas no objetivo e sua especificidade na promoção da saúde, prevenção de acidentes e doenças ocupacionais, reabilitação laboral e favorecimento da populaçáo trabalhadora.

Palavras-chave: Gestão de Riscos, Saúde do Trabalhador, Promoção da Saúde, Impacto Psicossocial, Serviços de Saúde Mental, Terapia Ocupacional.

\section{$\underline{\text { Abstract }}$}

Introduction: Psychosocial factors are dynamic interactions between different dimensions of the human being, task, occupation, environment, among others. The alteration of this dynamic produces risks that impact the health and well-being of workers. Objective: To identify and propose intervention strategies focused on psychosocial factors at the occupational level from occupational therapy Method: Study of qualitative, transversal and hermeneutical historical paradigm. Two phases focused on psychosocial interventions of occupational origin from occupational therapy. The first phase of documental search and the second, a survey of Colombian occupational therapists about their specific experience in psychosocial risk intervention. Analysis are carried out by triangulation. Results: Seven documents with theoretical, practical and historical influence relevant to the investigation and subsequent analysis are identified. From the second phase, 11 
responses from Colombian occupational therapists are validated, which describe two questions of interest for this investigation related to protective or preventive factors regarding psychosocial risks of work origin. About the second question describe actions, interventions or activities that your professional expertise has considered effective for the formulation of a psychosocial intervention strategy. An analytical triangulation is carried out where strategies derived from the documentary search, the answers of the survey and the compiled information are proposed. Categories of promotion, prevention, theory and support are also identified. Conclusion: Thirteen intervention strategies are proposed framed in the objective and its specificity in health promotion, prevention of accidents and occupational diseases, labor rehabilitation and favoring the working population.

Keywords: Risk Management, Occupational Health, Health Promotion, Psychosocial Impact, Mental Health Services, Occupational Therapy.

\section{Introducción}

Los factores psicosociales son concebidos como interacciones de la relación dinámica del trabajo, producción, ambiente, percepciones y capacidades del trabajador (Kalimo et al., 1987). Cuando estos tienen la probabilidad de afectar de forma negativa a la salud y al bienestar del trabajador se convierten en factores de riesgo (Moreno Jiménez, 2011).

En Colombia, la normatividad ha buscado identificar, evaluar, prevenir, intervenir y monitorear los factores de riesgo psicosocial. Es el caso de la resolución 2646 de 2008 la cual dispuso estas acciones y, en el proceso de identificar estos factores, ha involucrado el espacio del trabajador y de su actividad productiva. En ese sentido, se han evidenciado categorías relevantes como: 1) Gestión organizacional (estilos de mando, pago y contrato), 2) Características de la organización del trabajo (demandas de la labor), 3) Características del grupo social del trabajo (trabajo en equipo y relaciones laborales), 4) Condiciones de la tarea (carga mental y emocional), 5) Interfase persona-tarea (dinámica de la relación laboral y el desempeño) y 6) jornada de trabajo (tiempos y ejecuciones) (Icontec International, 2010; Colombia, 2007, 2008, 2015b).

Posteriormente, con la expedición de la Ley 1616 de 2013 de Salud Mental, se reconocen otros factores de riesgo psicosocial presentes en el trabajo como lo son los intralaborales, extralaborales e individuales los cuales incluyen: 1) Carga física (esfuerzo fisiológico que demanda la ocupación), 2) Medioambiente de trabajo (aspectos físicos), 3) Uso del tiempo libre, 4) Transporte casa - trabajo y viceversa, 5) Redes de apoyo, 6) Acceso a servicios de salud, 7) Características sociodemográficas, 8) Características de personalidad y estilos de afrontamiento, y 9) Condiciones de salud (evaluadas por medio de los exámenes ocupacionales) (Colombia, 2013).

Por otro lado, las diferentes correlaciones entre los accidentes y enfermedades de origen laboral con el desarrollo paralelo de enfermedades de origen mental determinaron que en la I Encuesta Nacional de Condiciones de Seguridad y Salud en el Trabajo en el Sistema General de Riesgos Laborales se indagara por las alteraciones mentales en el trabajo. Entre estas se evidenciaron la depresión (56\%), ansiedad (9\%) y, en menor 
medida, trastornos de adaptación, afectivos y del comportamiento (7-5\%) (Colombia, 2007). Subsiguientemente, la II Encuesta Nacional permitió reconocer que los factores de riesgo psicosocial tienen impacto en la salud y el bienestar de los trabajadores y, que diferentes enfermedades de origen laboral parten o están relacionadas con enfermedades mentales, dentro de estas relaciones se identificaron algunas actividades que pueden desencadenar una alteración mental, entre ellas: atención a usuarios (60\%), mantener un nivel de atención alto o muy alto (47.4\%), atender varias tareas al mismo tiempo (36\%), trabajo monótono (30.6\%), carga emocional (15\%) y percepción de la labor compleja o difícil (10\%) (Colombia, 2015a).

Producto de las diferentes encuestas y normas que se han desarrollado en el marco de esta problemática, actualmente, y en búsqueda de garantizar la promoción de la salud y la prevención del trastorno mental en ámbitos laborales, la Resolución 2404 de 2019 incluye la evaluación, intervención y monitoreo de los riesgos psicosociales en todos los ambientes de trabajo. También contempla las evaluaciones oportunas para determinar a tiempo, proteger y/o recuperar la salud mental alterada por las exposiciones relacionadas con los factores psicosociales y sus coadyuvantes (Colombia, 2019).

Partiendo de lo anteriormente mencionado, la intervención de los factores de riesgo psicosocial relacionados con el trabajo toma relevancia al involucrar diferentes disciplinas entre ellas la Terapia Ocupacional, buscando en primera medida cumplir con los diferentes compromisos y acuerdos internacionales como el Convenio 161 de 1985 de la Organización Internacional del Trabajo y la decisión 584 de 2004 de la Comunidad Andina. Estas disposiciones y las normatividades nacionales establecen que los empleadores se encuentran en la responsabilidad de crear y conservar un medio ambiente de trabajo seguro y sano que favorezca la salud e integridad física y mental de los trabajadores, partiendo desde la adaptación del trabajo a las capacidades de estos y teniendo en cuenta la ergonomía, la productividad y el bienestar (Comunidad Andina de Naciones, 2004; Organización Internacional del Trabajo, 1985). Otro punto de relevancia para la intervención radica en las cifras de accidentes y/o enfermedades de trabajo las cuales ya superan las 6,400 personas afectadas al día (Organización Internacional del Trabajo, 2019) configurando los factores psicosociales de origen laboral como de primera intervención, alarmantes y, de efecto directo sobre la salud general (Leka \& Jain, 2010; Marmot et al., 2012).

Es en ese sentido que la Terapia Ocupacional históricamente ha planteado y generado propuestas de atención que buscan facilitar el desempeño ocupacional de la persona; como referente Latinoamericano, la Terapia Ocupacional Psicosocial, contempla epistemológica y conceptualmente que la intervención trasciende de los modelos biomédicos para centrarse en perspectivas biopsicosociales (Paganizzi, 2007) permitiendo así una intervención integral que tenga como valor el significado de la ocupación y el bienestar en el quehacer laboral. Otras experiencias identificadas con participación desde Terapia Ocupacional son: 1) Intervención y/o prevención del Burnout con rutinas de descompresión laboral, mejora del estado físico con ejercicio, relajación, buenos hábitos, manejo del tiempo y participación en actividades de tiempo libre (Vidal Sánchez, 2015); 2) Procesos de reincorporación laboral con trabajadores que presentan Trastorno de Estrés Postraumático para facilitar el acceso a los distintos recursos laborales y adaptarlos a las necesidades individuales (Munar, 2017); 3) Evaluación del riesgo psicosocial a nivel intralaboral y extralaboral para así llevar a 
cabo un programa de bienestar ocupacional (Moreno \& Báez, 2010); 4) Intervención para la prevención de factores de riesgo psicosocial en docentes de colegios (Espejo et al., 2014) y, 5) la Intervención con empleadores sobre cómo entrenar hábitos psicosociales laborales que faciliten la creación y apropiación de una cultura organizacional de autocuidado (Gil et al., 2016).

De acuerdo con este contexto, se identifica que las intervenciones desde Terapia Ocupacional convergen en la implementación de estrategias orientadas al logro de un equilibrio ocupacional en donde la persona entra en contacto con la ocupación y el ambiente para establecer mecanismos de resiliencia que a su vez se ajustan dependiendo de los hábitos, rutinas y roles que desempeña en los diferentes entornos de interacción laboral (Kielhofner, 2002; Law et al., 1996).

En búsqueda de ese equilibrio ocupacional en trabajadores, entendiendo las complejidades de la relación con el ambiente-trabajo y a partir de la conceptualización normativa en Colombia, es objetivo del presente estudio identificar y proponer estrategias de intervención enfocadas en los factores psicosociales a nivel laboral desde terapia ocupacional que contribuyan en la promoción de la salud, prevención de los accidentes y enfermedades laborales, dirijan la rehabilitación laboral y permitan el favorecimiento del bienestar de la población trabajadora.

\section{Metodología}

Estudio de enfoque cualitativo, de tipo transversal y bajo un paradigma histórico hermenéutico. La investigación se desarrolló en dos fases: la primera a través de una búsqueda documental para análisis temático sobre estrategias de intervención psicosocial laboral específica en Terapia Ocupacional y, la segunda, el diseño de una encuesta con miras a identificar experiencias de intervención psicosocial de terapeutas ocupacionales colombianos en el área laboral. Finalmente, se realizó un análisis por triangulación con el fin de consolidar la información documental y experiencial en pro de las estrategias de intervención.

Fase I: se realizó una búsqueda documental enfocada en libros y documentos históricos, de fundamentación y de desarrollo profesional de la Terapia Ocupacional. Como criterios de inclusión se tuvieron en cuenta aquellos que: 1) su enfoque temático incluyera Terapia Ocupacional, 2) en idioma original español, inglés y portugués; y, 3) se incluyeran aspectos de prevención, evaluación y/o intervención de enfermedades mentales enmarcadas en el área laboral. El rastreo documental se llevó a cabo en los portales y repositorios de universidades; además de bases de datos especializadas como Directory of Open Access Books (DOAB), OT Seeker, Elsevier Health, IEE Wiley Books y Springer Books.

Fase II: En cuanto al cuestionario, este fue de carácter semiestructurado, en línea y disponible para revisión durante los meses del estudio. Este cuestionario fue validado por docentes pertenecientes a la Universidad Nacional de Colombia quienes efectuaron una revisión y prueba piloto. El cuestionario fue dirigido a terapeutas ocupacionales residentes en Colombia que se desempeñaran en las áreas sociolaboral (trabajo, industria, laboral) y psicosocial (salud mental).

La recolección de datos se llevó a cabo por medio de un cuestionario virtual soportado por la Universidad Nacional de Colombia. Este cuestionario fue difundido 
por correo electrónico de forma directa a terapeutas ocupacionales en Colombia según bases de datos preexistentes y se utilizaron otros medios de difusión como el Colegio Colombiano de Terapia Ocupacional (organización gremial de terapeutas ocupacionales colombianos) que a su vez los distribuyó a los profesionales agremiados. Como criterios de inclusión y validación para ingreso al estudio se tuvieron en cuenta: 1) profesionales en Terapia Ocupacional, 2) residentes en Colombia, 3) que su experiencia profesional fuese en las áreas laboral y/o de salud mental superior a 5 años, 4) que haya diligenciado la encuesta en su totalidad y 5) que haya aceptado la participación en la investigación.

\subsection{Consideraciones éticas}

La investigación fue avalada por el Comité de ética de la Facultad de Medicina de la Universidad Nacional de Colombia para su ejecución bajo el acta $\mathrm{N}^{\circ}$ 006-060-17 del 27 de abril de 2017. La recolección de datos por medio del cuestionario virtual se llevó a cabo previa autorización de los profesionales por medio de un consentimiento informado y aceptación expresa de participación.

\section{Resultados}

Fase I: la revisión permitió identificar 7 documentos publicados con teorías, experiencias y recopilaciones históricas que evidenciaban la intervención de terapia ocupacional psicosocial en el área laboral Tabla 1.

Tabla 1. Información de documentos recopilados y su relación específica con la temática. Fuente: elaborado por autores con base en resultados del estudio.

\begin{tabular}{|c|c|c|}
\hline Documento & Referencia & Relación \\
\hline $\begin{array}{l}\text { Terapia Ocupacional en salud mental: } \\
\text { principios y práctica }\end{array}$ & (Molina \& Arnaiz, 1998) & $\begin{array}{l}\text { - Teórica } \\
\text { - Práctica }\end{array}$ \\
\hline $\begin{array}{l}\text { Conceptos fundamentales de Terapia } \\
\text { Ocupacional }\end{array}$ & (Polonio et al., 2001) & \multirow{3}{*}{$\begin{array}{l}\text { - Teórica } \\
\text { - Práctica } \\
\text { - Histórica }\end{array}$} \\
\hline $\begin{array}{l}\text { Terapia Ocupacional: conocimiento y } \\
\text { práctica en Colombia }\end{array}$ & (Trujillo, 2002) & \\
\hline $\begin{array}{c}\text { Modelo de la Ocupación Humana: Teoría } \\
\text { y aplicación }\end{array}$ & (Kielhofner, 2004) & \\
\hline $\begin{array}{l}\text { Terapia Ocupacional en discapacitados } \\
\text { físicos: Teoría y práctica }\end{array}$ & (López, 2004) & \multirow{2}{*}{$\begin{array}{l}\text { - Teórica } \\
\text { - Práctica }\end{array}$} \\
\hline $\begin{array}{c}\text { Terapia Ocupacional intervención en } \\
\text { distintas patologías }\end{array}$ & (Rincón Herrera et al., 2007). & \\
\hline Willard \& Spackman Terapia Ocupacional & (Shell et al., 2016) & $\begin{array}{l}\text { - Teórica } \\
\text { - Práctica } \\
\text { - Histórica }\end{array}$ \\
\hline
\end{tabular}

Fase II: Un total de 18 respuestas fueron consolidadas en el sistema. Posteriormente, estas se revisaron con el fin de identificar faltantes y consecución normal del cuestionario. En esta revisión se eliminaron 7 ya que no cumplían con 
los criterios de inclusión a la investigación. El resultado final fueron 11 respuestas válidas.

Dentro de los resultados de las repuestas validadas se precisarán dos ítems que permitieron la conducción de la investigación y, posteriormente, el análisis de esta. El primer ítem corresponde a la pregunta ¿Conoce usted algún factor protector o preventivo que evite o reduzca la probabilidad de daño de un riesgo psicosocial en el trabajo? Ante esto, el $100 \%$ de los encuestados respondió y diferenció los factores con los siguientes resultados Tabla 2.

Tabla 2. Factores protectivos o preventivos identificados desde los terapeutas ocupacionales en relación con los riesgos laborales. Fuente: elaborado por autores con información del estudio.

\section{Factores}

\begin{tabular}{|c|c|c|c|}
\hline & Intralaboral & Extralaboral & Personal \\
\hline Acciones & $\begin{array}{c}\text { - Pausas activas } \\
\text { - Salario adecuado (formación y } \\
\text { experiencia) } \\
\text { - Reconocimientos } \\
\text { - Horarios adecuados } \\
\text { - Ambiente laboral } \\
\text { - Relaciones interpersonales } \\
\text { - Programas de bienestar laboral } \\
\text { - Estilos de vida saludable } \\
\text { - Buen diseńo del puesto de trabajo } \\
\text { - Contar con profesionales } \\
\text { sociosanitarios y psicosociales. } \\
\text { - Estrategias de ergonomía cognitiva } \\
\text { - Comité de convivencia laboral } \\
\text { - Tareas acordes a las capacidades } \\
\text { - Posibilidad de avanzar laboralmente } \\
\text { - Participación en Seguridad y Salud en } \\
\text { el Trabajo }\end{array}$ & $\begin{array}{c}\text { - No llevar trabajo a } \\
\text { casa } \\
\text { - Fomentar la } \\
\text { participación en } \\
\text { ambientes sociales }\end{array}$ & $\begin{array}{l}\text { - Contar con una } \\
\text { buena red de apoyo } \\
\text { - Buena autoestima }\end{array}$ \\
\hline
\end{tabular}

El segundo ítem corresponde a la pregunta: ¿podría describir alguna(s) intervenciones o acciones que haya realizado como terapeuta ocupacional dentro de su experiencia en el área laboral y/o de salud mental enmarcado en riesgos psicosociales en el trabajo? Como resultado, el 100\% de los encuestados respondió y posterior a un análisis del grupo investigador, se agruparon los datos en la Tabla 3. 
Tabla 3. Respuestas agrupadas, identificación entre prevención/promoción y frecuencia de referencia entre los terapeutas ocupacionales. Fuente: elaborado por autores con información del estudio.

\begin{tabular}{|c|c|c|}
\hline $\begin{array}{l}\text { Riesgo mitigado (prevención) // } \\
\text { Favorecimiento (promoción) } \\
\end{array}$ & Acción / intervención / Actividad & $\begin{array}{c}\text { Frecuencia de } \\
\text { respuesta }\end{array}$ \\
\hline $\begin{array}{c}\text { Favorecimiento: } \\
\text { - Manejo del tiempo } \\
\text { - Trabajo por horario } \\
\text { - Apoyo social en organización }\end{array}$ & $\begin{array}{l}\text { - Distribución del tiempo } \\
\text { - Pausas activas } \\
\text { - Organización de rutinas } \\
\text { - Manejo del tiempo libre } \\
\text { - Actividad psicoeducativa }\end{array}$ & ALTA \\
\hline $\begin{array}{c}\text { Favorecimiento: } \\
\text { - Habilidades y destrezas } \\
\text { individuales } \\
\text {-Mecanismos de comunicación e } \\
\text { interacción }\end{array}$ & $\begin{array}{l}\text { - Valoración capacidad Vs. Demanda } \\
\text { - Habilidades de orientación } \\
\text { - Actividad de fomento de talentos } \\
\text { - Estilos de vida y trabajo saludable } \\
\text { - Matriz de comunicación }\end{array}$ & ALTA \\
\hline $\begin{array}{l}\text { Mitigación del riesgo: } \\
\text { - Ansiedad y estrés laboral } \\
\text { - Asistencia al trabajador }\end{array}$ & $\begin{array}{l}\text { - Actividad de relajación } \\
\text { - Actividad psicoeducativa } \\
\text { - Orientación ocupacional }\end{array}$ & ALTA \\
\hline $\begin{array}{c}\text { Favorecimiento: } \\
\text { - Fomento actividad educativa, } \\
\text { deportiva, recreativa y cultura }\end{array}$ & $\begin{array}{l}\text { - Actividad psicomotora } \\
\text { - Actividad psicoeducativa } \\
\text { - Salidas extramurales }\end{array}$ & MODERADA \\
\hline $\begin{array}{l}\text { Favorecimiento: } \\
\text { - Espacio extralaboral }\end{array}$ & $\begin{array}{c}\text { - Actividad familiar } \\
\text { - Actividad psicoeducativa } \\
\text { - Actividad de autocuidado, hábitos y roles }\end{array}$ & MODERADA \\
\hline $\begin{array}{l}\text { Mitigación: } \\
\text { - Alteraciones persona-trabajo } \\
\text { - Mecanismo de carga de trabajo }\end{array}$ & $\begin{array}{c}\text { - Valoración salud laboral } \\
\text { - Recomendaciones ocupacionales } \\
\text { - Análisis de puestos de trabajo } \\
\text { - Asesoría en educación } \\
\text { - Actividades psicoeducativas }\end{array}$ & MODERADA \\
\hline $\begin{array}{l}\text { Favorecimiento: } \\
\text { - Desarrollo de resiliencia } \\
\text { - Técnicas de afrontamiento }\end{array}$ & $\begin{array}{l}\text { - Actividad socioafectiva } \\
\text { - Actividades de comunicación }\end{array}$ & BAJA \\
\hline $\begin{array}{l}\text { Mitigación del riesgo: } \\
\text { - Consumo de sustancias }\end{array}$ & $\begin{array}{l}\text { - Campaña de prevención y atención en el } \\
\text { consumo de sustancias }\end{array}$ & BAJA \\
\hline $\begin{array}{c}\text { Favorecimiento: } \\
\text { - Preparación para la pensión o } \\
\text { jubilación } \\
\text { - Gestión del cambio }\end{array}$ & $\begin{array}{l}\text { - Orientaciones de seguridad y atención en el } \\
\text { sistema de salud. } \\
\text { - Actividad psicoeducativa } \\
\text { - Actividad proyecto de vida }\end{array}$ & BAJA \\
\hline $\begin{array}{c}\text { Favorecimiento: } \\
\text { - Formación de los trabajadores } \\
\text { - Inducción y reinducción }\end{array}$ & $\begin{array}{l}\text { - Actividades psicoeducativas } \\
\text { - Apoyos en diseño y acompañamiento } \\
\text { - Planes de capacitación }\end{array}$ & BAJA \\
\hline $\begin{array}{l}\text { Favorecimiento: } \\
\text { - Promoción de la salud mental }\end{array}$ & $\begin{array}{l}\text { - Actividad cultural y recreativa } \\
\text { - Reconocimiento de derechos } \\
\text { - Creación de proyectos participativos }\end{array}$ & BAJA \\
\hline $\begin{array}{l}\text { Mitigación del riesgo: } \\
\text { - Otras alteraciones o } \\
\text { enfermedades mentales }\end{array}$ & $\begin{array}{l}\text { - Actividades empresa-familia } \\
\text { - Seguimiento clínico } \\
\text { - Reincorporación laboral }\end{array}$ & BAJA \\
\hline $\begin{array}{l}\text { Enriquecimiento de puestos de } \\
\text { trabajo }\end{array}$ & $\begin{array}{l}\text { - Evaluaciones de puesto } \\
\text { - Entrega de materiales, equipos y dotaciones } \\
\text { - Organización de tareas y ambiente }\end{array}$ & BAJA \\
\hline
\end{tabular}




\subsection{Análisis por triangulación}

Con los resultados obtenidos de la investigación documental y de los cuestionarios en línea se realizó un análisis de datos Tabla 4. Para esta, se pone en primer orden la estrategia propuesta y por ende, la primera puesta en común desde lo teórico-práctico. Posteriormente, se postularon las siguientes categorías con el fin de complementar la estrategia y tener un sustento teórico amplio. Las categorías fueron: 1) Acciones de promoción de la salud, 2) prevención de la enfermedad y de accidentes de trabajo, y 3) Herramientas teórico-prácticas.

Tabla 4. Triangulación con análisis/comparación de los datos obtenidos por búsqueda y cuestionario. Fuente: elaborado por autores con información del estudio.

\begin{tabular}{|c|c|c|c|c|}
\hline \multicolumn{5}{|c|}{ Enmarcado en los riesgos psicosociales de origen laboral } \\
\hline $\begin{array}{l}\text { Estrategia } \\
\text { propuesta }\end{array}$ & $\begin{array}{c}\text { Acciones de } \\
\text { promoción de la } \\
\text { salud }\end{array}$ & $\begin{array}{l}\text { Intervenciones de } \\
\text { prevención de } \\
\text { enfermedades y } \\
\text { accidentes }\end{array}$ & $\begin{array}{l}\text { Herramientas } \\
\text { teórico - prácticas }\end{array}$ & $\begin{array}{c}\text { Supuesto } \\
\text { teórico, } \\
\text { documental o } \\
\text { histórico }\end{array}$ \\
\hline $\begin{array}{l}\text { Ocupación } \\
\text { significativa }\end{array}$ & $\begin{array}{l}\text { - Fomento de } \\
\text { actividades } \\
\text { educativas, } \\
\text { deportivas, } \\
\text { recreativas y } \\
\text { culturales. } \\
\text { - Fomento de } \\
\text { estilos de vida } \\
\text { saludable. }\end{array}$ & $\begin{array}{l}\text { - Actividades de } \\
\text { preparación para la } \\
\text { pensión. } \\
\text { - Prevención del } \\
\text { consumo de sustancias } \\
\text { psicoactivas. }\end{array}$ & $\begin{array}{l}\text { Modificación de } \\
\text { conductas } \\
\text { ocupacionales a } \\
\text { través de: } \\
\text { - Modelo de } \\
\text { creencias en salud. } \\
\text { - Modelo de } \\
\text { estadios del cambio. } \\
\text { - Teoría cognitiva } \\
\text { social. }\end{array}$ & (Reitz, 2016) \\
\hline Adaptación & $\begin{array}{l}\text { - Desarrollo de } \\
\text { estrategias de } \\
\text { afrontamiento. }\end{array}$ & $\begin{array}{l}\text { - Gestión del cambio. } \\
\text { - Gestión del trabajo } \\
\text { por turnos. } \\
\text { - Actividades de } \\
\text { preparación para la } \\
\text { pensión. } \\
\text { - Desarrollo y } \\
\text { fortalecimiento de la } \\
\text { resiliencia en el } \\
\text { ambiente laboral. }\end{array}$ & $\begin{array}{l}\text { Uso de habilidades } \\
\text { adaptativas a partir } \\
\text { de: } \\
\text { - Teoría de } \\
\text { adaptación } \\
\text { ocupacional. } \\
\text { - Modelo de } \\
\text { habilidades } \\
\text { adaptativas. }\end{array}$ & $\begin{array}{l}\text { (Rincón } \\
\text { Herrera et al., } \\
\text { 2007) }\end{array}$ \\
\hline $\begin{array}{l}\text { Resolución de } \\
\text { problemas }\end{array}$ & $\begin{array}{l}\text { - Servicio de } \\
\text { asistencia al } \\
\text { trabajador. } \\
\text { - Mejoramiento } \\
\text { participativo de las } \\
\text { condiciones } \\
\text { psicosociales del } \\
\text { trabajo. }\end{array}$ & $\begin{array}{l}\text { - Gestión del cambio. } \\
\text { - Mejoramiento } \\
\text { participativo de las } \\
\text { condiciones } \\
\text { psicosociales del trabajo. }\end{array}$ & $\begin{array}{l}\text { Centrada en las } \\
\text { habilidades de } \\
\text { aprendizaje, el } \\
\text { proceso de } \\
\text { aprendizaje y } \\
\text { criterios cognitivos: } \\
\text { - Teoría de } \\
\text { aprendizaje social. } \\
\text { - Teoría } \\
\text { constructivista. } \\
\text { - Análisis de la } \\
\text { actividad. }\end{array}$ & $\begin{array}{c}\text { (Helfrich, } \\
2016 ; \\
\text { Polonio et al., } \\
2001)\end{array}$ \\
\hline
\end{tabular}


Tabla 4. continuación

\begin{tabular}{|c|c|c|c|c|}
\hline $\begin{array}{l}\text { Análisis de } \\
\text { puesto de } \\
\text { trabajo }\end{array}$ & $\begin{array}{l}\text { - Diseño y } \\
\text { fortalecimiento del } \\
\text { plan de formación } \\
\text { de los trabajadores. }\end{array}$ & $\begin{array}{l}\text { - Inducción y } \\
\text { reinducción. } \\
\text { - Claridad del rol como } \\
\text { pilar del desempeño. } \\
\text { - Construcción del } \\
\text { ajuste persona - trabajo. } \\
\text { - Rotación de puestos } \\
\text { de trabajo. }\end{array}$ & $\begin{array}{l}\text { - Descripción de } \\
\text { puesto de trabajo: } \\
\text { funciones, } \\
\text { responsabilidades, } \\
\text { experiencia y } \\
\text { habilidad. } \\
\text { - Análisis de las } \\
\text { tareas: demandas } \\
\text { físicas, cognitivas y } \\
\text { psicológicas, }\end{array}$ & $\begin{array}{c}\text { (King \& } \\
\text { Olson, 2016) }\end{array}$ \\
\hline $\begin{array}{c}\text { Rehabilitación } \\
\text { integral }\end{array}$ & $\begin{array}{l}\text { - Promoción de la } \\
\text { salud mental y } \\
\text { prevención del } \\
\text { trastorno mental en } \\
\text { el trabajo. }\end{array}$ & $\begin{array}{l}\text { - Promoción de la salud } \\
\text { mental y prevención del } \\
\text { trastorno mental en el } \\
\text { trabajo }\end{array}$ & $\begin{array}{l}\text { - Rehabilitación } \\
\text { funcional. } \\
\text { - Rehabilitación } \\
\text { profesional. } \\
\text { - Rehabilitación } \\
\text { social. }\end{array}$ & $\begin{array}{l}\text { (Scaffa, } \\
\text { 2016a; } \\
\text { Vaquero, } \\
\text { 2011) }\end{array}$ \\
\hline $\begin{array}{l}\text { Regulación } \\
\text { emocional }\end{array}$ & $\begin{array}{l}\text { - Desarrollo de } \\
\text { estrategias de } \\
\text { afrontamiento. }\end{array}$ & $\begin{array}{l}\text { - Actividades de } \\
\text { preparación para la } \\
\text { pensión. }\end{array}$ & $\begin{array}{l}\text { - Evaluación de } \\
\text { antecedentes sociales } \\
\text { y ocupacionales, } \\
\text { habilidades } \\
\text { emocionales } \\
\text { - Intervención: } \\
\text { manejo de } \\
\text { emociones e } \\
\text { inteligencia } \\
\text { emocional }\end{array}$ & $\begin{array}{l}\text { (Scaffa, } \\
\text { 2016b) }\end{array}$ \\
\hline $\begin{array}{c}\text { Fomento de } \\
\text { intereses }\end{array}$ & $\begin{array}{l}\text { - Fomento de } \\
\text { actividades } \\
\text { educativas, } \\
\text { deportivas, } \\
\text { recreativas y } \\
\text { culturales. }\end{array}$ & $\begin{array}{l}\text { - Aprovechamiento } \\
\text { integral de habilidades y } \\
\text { destrezas individuales. } \\
\text { - Enriquecimiento de } \\
\text { puestos de trabajo. } \\
\text { - Actividades de } \\
\text { preparación para la } \\
\text { pensión. }\end{array}$ & $\begin{array}{l}\text { - Evaluación: } \\
\text { Listado de Intereses. } \\
\text { - Intervención: } \\
\text { Programas para } \\
\text { educar a las personas } \\
\text { sobre el ocio, los } \\
\text { recursos personales y } \\
\text { comunitarios. }\end{array}$ & $\begin{array}{l}\text { (Kielhofner, } \\
\text { 2004; } \\
\text { Primeau, } \\
\text { 2016) }\end{array}$ \\
\hline $\begin{array}{c}\text { Equilibrio } \\
\text { ocupacional }\end{array}$ & & $\begin{array}{l}\text { - Conciliación de } \\
\text { entornos intra y } \\
\text { extralaboral }\end{array}$ & $\begin{array}{l}\text { - Evaluación: } \\
\text { Cuestionario } \\
\text { Ocupacional } \\
\text { - Intervención: } \\
\text { Programas dirigidos } \\
\text { a la identificación de } \\
\text { problemas y } \\
\text { soluciones para tener } \\
\text { equilibrio } \\
\text { ocupacional }\end{array}$ & $\begin{array}{l}\text { (Ávila } \\
\text { Álvarez et al., } \\
\text { 2010; } \\
\text { Kielhofner, } \\
\text { 2004) }\end{array}$ \\
\hline $\begin{array}{l}\text { Hábitos y } \\
\text { rutinas }\end{array}$ & $\begin{array}{l}\text { - Fomento de } \\
\text { estilos de vida } \\
\text { saludable. }\end{array}$ & $\begin{array}{l}\text { - Gestión de cargas de } \\
\text { trabajo. } \\
\text { - Gestión de pausas en } \\
\text { el trabajo. }\end{array}$ & $\begin{array}{l}\text { - Evaluación: } \\
\text { Cuestionario } \\
\text { Ocupacional } \\
\text { - Intervención: } \\
\text { Actividades que } \\
\text { favorezcan generar } \\
\text { nuevos hábitos y } \\
\text { rutinas }\end{array}$ & $\begin{array}{c}\text { (Polonio et al., } \\
\text { 2001) }\end{array}$ \\
\hline
\end{tabular}


Tabla 4. continuación

\begin{tabular}{|c|c|c|c|c|}
\hline $\begin{array}{c}\text { Perfil } \\
\text { ocupacional }\end{array}$ & $\begin{array}{l}\text { - Prevención del } \\
\text { consumo de } \\
\text { sustancias } \\
\text { psicoactivas. }\end{array}$ & $\begin{array}{l}\text { - Prevención del } \\
\text { consumo de sustancias } \\
\text { psicoactivas. }\end{array}$ & $\begin{array}{l}\text { Recolección de } \\
\text { información de la } \\
\text { historia ocupacional } \\
\text { y experiencias de la } \\
\text { persona. } \\
\text { Patrones de vida, } \\
\text { intereses, valores, } \\
\text { necesidades y } \\
\text { proyección } \\
\text { ocupacional }\end{array}$ & $\begin{array}{c}\text { (Ávila } \\
\text { Álvarez et al., } \\
\text { 2010; Peñas } \\
\text { Felizzola, } \\
\text { 2005) }\end{array}$ \\
\hline $\begin{array}{l}\text { Orientación } \\
\text { vocacional / } \\
\text { ocupacional }\end{array}$ & $\begin{array}{l}\text { - Servicio de } \\
\text { asistencia al } \\
\text { trabajador. } \\
\text { - Información sobre } \\
\text { temas de salud, } \\
\text { pensión, vivienda, } \\
\text { educación, finanzas } \\
\text { familiares y cajas de } \\
\text { compensación. }\end{array}$ & $\begin{array}{l}\text { - Información sobre } \\
\text { temas de salud, pensión, } \\
\text { vivienda, educación, } \\
\text { finanzas familiares y } \\
\text { cajas de compensación. } \\
\text { - Actividades de } \\
\text { preparación para la } \\
\text { pensión. }\end{array}$ & $\begin{array}{l}\text { - Desarrollo de } \\
\text { procesos grupales e } \\
\text { individuales que } \\
\text { incluyan perfilar } \\
\text { intereses y objetivos } \\
\text { personales }\end{array}$ & $\begin{array}{l}\text { (Polonio et al., } \\
\text { 2001) }\end{array}$ \\
\hline Autocuidado & $\begin{array}{l}\text { - Fomento de } \\
\text { estilos de vida } \\
\text { saludable. } \\
\text { - Prevención del } \\
\text { consumo de } \\
\text { sustancias } \\
\text { psicoactivas. }\end{array}$ & $\begin{array}{l}\text { - Gestión del trabajo } \\
\text { por turnos. } \\
\text { - Prevención del } \\
\text { consumo de sustancias } \\
\text { psicoactivas. }\end{array}$ & $\begin{array}{l}\text { - Evaluación: } \\
\text { Entrevistas, } \\
\text { cuestionarios, } \\
\text { autoinformes u } \\
\text { observación directa } \\
\text { de la persona } \\
\text { - Intervención: } \\
\text { Programas de } \\
\text { bienestar } \\
\text { ocupacional y } \\
\text { promoción de estilos } \\
\text { de vida saludable }\end{array}$ & $\begin{array}{l}\text { (Polonio et al., } \\
\text { 2001) }\end{array}$ \\
\hline $\begin{array}{l}\text { Sueño y } \\
\text { descanso }\end{array}$ & $\begin{array}{l}\text { - Fomento de } \\
\text { estilos de vida } \\
\text { saludable. }\end{array}$ & $\begin{array}{l}\text { - Gestión del trabajo } \\
\text { por turnos. }\end{array}$ & $\begin{array}{l}\text { - Evaluación: Escala } \\
\text { de somnolencia de } \\
\text { Epworth, Diario de } \\
\text { horas de sueńo. } \\
\text { - Intervención: } \\
\text { Programas de } \\
\text { bienestar } \\
\text { ocupacional } \\
\text { dirigidos a hábitos y } \\
\text { conductas que } \\
\text { influyen sobre el } \\
\text { sueño, esquema del } \\
\text { sueño en el ciclo } \\
\text { vital, manejo de } \\
\text { descansos en horario } \\
\text { laboral. }\end{array}$ & (Solet, 2016) \\
\hline
\end{tabular}




\section{Discusión}

En el presente estudio se construyen un conjunto de ideas estructuradas a partir de fundamentos teóricos, teórico-prácticos, históricos, experienciales y vivenciales cuyo fin es brindar herramientas y sustento al terapeuta ocupacional en su intervención ante factores de riesgo psicosocial en un ambiente laboral (Ávila Álvarez et al., 2010). Por ello, se traen a discusión normatividades nacionales e internacionales que por un lado develan la necesidad de identificar e intervenir factores que derivan de la psiquis humana y que por otro, se ponen en juego en el quehacer y la ocupación como el trabajo (Icontec International, 2010; Kalimo et al., 1987; Leka \& Jain, 2010; Marmot et al., 2012).

En Colombia, diferentes documentos han evidenciado la relación entre los riesgos psicosociales, sus alteraciones y las características del trabajo como la organización, las tareas, los grupos sociales, la relación de trabajo y capacidad, entre otras; estas relaciones ahora se ven más claras con las estadísticas, ya que estas permiten divisar un panorama de alteraciones mentales, comportamentales y actitudinales relacionadas directamente con el trabajo (Icontec International, 2010; Colombia, 2007, 2013, 2015b).

Partiendo de los resultados expuestos, se identifica que las estrategias de Terapia Ocupacional históricamente han propuesto una intervención diferente y quizá alternativa en la forma de abordar los factores de riesgo psicosocial en el área laboral; tanto desde un enfoque de rehabilitación integral como desde un ámbito preventivo. Entender las dinámicas propuestas desde el desempeño ocupacional, la salud, el bienestar y la satisfacción de la ocupación es un proceso volátil y multivariable que deriva no sólo en acciones basadas en la alteración, accidente o enfermedad, sino el introducir actividades comunes con sentido, significado y relevancia para la persona permitiendo que con ello su calidad de vida mejore y estos riesgos desde el trabajo sean de probabilidad mínima o nula (Reason, 1997; Romero Ayuso, 2010). Sin embargo, se ha evidenciado que las intervenciones comunes en el área laboral tienden a ser acompañadas de acciones comunes realizadas desde la psiquiatría y psicología recurriendo a un círculo común que no favorece el cambio o transformación del área y por ello, continua el aumento de las cifras de accidentes y enfermedades (Kalimo et al., 1987; Organización Internacional del Trabajo, 2019; Paganizzi, 2007; Paterson, 2008). En este estudio se demuestra la relación no reduccionista de la Terapia Ocupacional al entender que, una vez se compensan las consecuencias de la enfermedad se siguen presentando una gran cantidad de limitaciones para el desempeño personal, ocupacional y social (Paganizzi, 2007).

Es importante validar y correlacionar las evidencias extraídas de los documentos recopilados con objetivo planteado en este documento, en donde efectivamente los terapeutas ocupacionales colombianos plantearon acciones, intervenciones o actividades en las áreas laboral y/o salud mental, denotando un manejo de diferentes herramientas enmarcadas en la salud, el bienestar y la concepción socio-humanística de las personas (Ávila Álvarez et al., 2010; Gutiérrez-Bolaño et al., 2019; Organización Mundial de La Salud, 1986, 2019; Peñas Felizzola, 2005; Trujillo, 2002).

Específicamente en este estudio se identificó una mezcla de instrucciones, ideas, teorías y paradigmas que involucraban la forma o la orientación de cómo se debe hacer la intervención desde terapia ocupacional psicosocial en el área laboral (Kielhofner, 
2004; López, 2004; Molina \& Arnaiz, 1998; Polonio et al., 2001; Rincón Herrera et al., 2007; Shell et al., 2016; Trujillo, 2002). Se evidenció que todos los documentos recolectados tienen una fundamentación teórica y práctica que permite validar las acciones; por otro lado, el $57 \%$ de estos documentos permite tener un seguimiento desde lo histórico hasta la realidad práctica actual del terapeuta ocupacional (Kielhofner, 2004; Polonio et al., 2001; Shell et al., 2016; Trujillo, 2002).

Finalmente, se evidencia una compilación y a modo de propuesta, de algunas estrategias que apuntan desde la concepción de una terapia ocupacional latinoamericana a un entendimiento de una dinámica ocupación, salud y productividad (Organización Mundial de La Salud, 2010; Peńas Felizzola, 2005; Trujillo, 2002). Estas propuestas se acercan a conceptos como los planteados por (O'Connor et al., 2014) en el sentido de interrelacionar una estrategia con: 1) unas acciones enmarcadas en la salud internacional (Organización Mundial de La Salud, 2010); 2) la prevención de la enfermedad/accidentes laborales (Gil-Monte, 2012) y 3) una sustentación teórica que impacta en las realidades laborales, psicosociales y actuales del país.

\section{Conclusión}

El presente estudio se llevó a cabo con el fin de identificar y proponer estrategias de intervención para los factores de riesgo psicosocial de origen laboral desde terapia ocupacional, lo cual derivó en las trece (13) estrategias develadas Véase Tabla 4. El proceso de recopilación de la información por medio de investigaciones y cuestionarios aplicados a terapeutas ocupacionales permite denotar que es reciente la participación de la terapia ocupacional en los factores psicosociales laborales.

Las estrategias derivadas del estudio se correlacionan no sólo con el objetivo general del estudio sino responden a un grupo de enfoques históricos y hermenéuticos los cuales se usarán y desarrollarán a modo de conclusión:

El primero, la contribución a la promoción de la salud, la cual se ve evidenciada en gran medida a partir de ocupaciones significativas involucrando el fomento de actividades educativas, deportivas, recreativas, culturales y, el desarrollo de acciones como la atención en los servicios de asistencia, la accesibilidad en servicios e información, el reforzamiento de los estilos de vida saludable, la atención emocional, entre otros.

El segundo, contribuciones a la prevención de los accidentes y enfermedades laborales, enfocado especialmente a: 1) adaptación laboral entendiendo la gestión del trabajo, sus horarios, el ambiente y las relaciones con las tareas; 2) el análisis de puestos de trabajo entendiendo las funciones, las estructuras corporales, el desempeño ocupacional y los ajustes requeridos; y 3) el fomento de intereses entendiendo las habilidades, destrezas, capacidades y el enriquecimiento del puesto de trabajo.

Adicionalmente, el tercero, contribuciones a la rehabilitación integral, a partir de la participación de actividades de tipo funcional, adaptación sociolaboral y entendiendo la salud mental, conociendo sus realidades, modificando los requerimientos de la labor y proyectando la integración laboral. Esto mediante la aplicación de evaluaciones ocupacionales domiciliarias, análisis ocupacional, análisis de exigencias, pruebas de trabajo, inspecciones y seguimientos. 
Finalmente, el cuarto, el favorecimiento del bienestar de la población trabajadora a través de un gran número de herramientas que incluyen modelos de creencias, percepciones, habilidades, destrezas, desempeños, entre otros. Si se sumaran las estrategias junto con los procesos reales de implementación a nivel empresarial se podría evidenciar que el bienestar de la población se mide en el impacto de estas en forma transversal a las dimensiones humanas.

Como limitaciones de la investigación se identificó: 1) la baja participación de terapeutas ocupacionales que aborden los factores psicosociales de origen laboral; 2) la escasa publicación de evidencia científica que aún desde escenarios clínicos, fortalecieran el ejercicio profesional; y 3) la dificultad para articular las áreas de salud mental y la laboral.

Es importante profundizar en la práctica de las estrategias de intervención de los factores de riesgo psicosocial acorde a las particularidades de una organización, de tal forma que se logre ampliar el reconocimiento de la terapia ocupacional en los ámbitos laborales con un enfoque psicosocial, no solo desde la teoría sino con la puesta en práctica desde sus múltiples intervenciones como lo son la promoción de la salud mental, la prevención de las enfermedades mentales y la rehabilitación integral.

\section{Agradecimientos}

A la terapeuta ocupacional Lilia Andrea Martínez Páez y al Departamento de la Ocupación Humana de la Universidad Nacional de Colombia, al Colegio Colombiano de Terapia Ocupacional (CCTO), Universidades y terapeutas ocupacionales quienes participaron en el estudio.

\section{Referencias}

Ávila Álvarez, A., Martínez Piédrola, R., Matilla Mora, R., Máximo Bocanegra, M., Méndez Méndez, B., \& Talavera Valverde, M. A. (2010). Marco de Trabajo para la práctica de la Terapia Ocupacional: Dominio y proceso. España: AOTA.

Colombia. Ministerio de la Protección Social. (2007). I Encuesta Nacional de Condiciones de Saludy Trabajo en el Sistema General de Riesgos Profesionales. Bogotá: Ministerio de La Protección Social.

Colombia. (2008, 17 de julio). Resolución 2646 de 2008. Por la cual se establecen disposiciones y se definen responsabilidades para la identificación, evaluación, prevención, intervención y monitoreo permanente de la exposición a factores de riesgo psicosocial en el trabajo. Diario Oficial, Bogotá.

Colombia. Congreso de la República. (2013, 21 de enero). Ley n. 1616, de 21 de enero de 2013. Por medio de la cual se expide la ley de Salud Mental y se dictan otras disposiciones. Diario Oficial $n^{o}$ 48.680, Colombia.

Colombia. Ministerio del Trabajo. (2015a). II Encuesta nacional de condiciones de seguridad y salud en el trabajo en el sistema general de riesgos. Bogotá: Ministerio del Trabajo.

Colombia. Ministerio del Trabajo. (2015b). Promoción, prevención e intervención de los factores psicosociales y sus efectos en población trabajadora: guía técnica general. Bogotá: Pontificia Universidad Javeriana.

Colombia. (2019). Resolución no 2404 de 22 de julio de 2019. Por la cual se adopta la Batería de Instrumentos para la Evaluación de Factores de Riesgo Psicosocial, la Guía Técnica General para la Promoción, Prevención e Intervención de los Factores Psicosociales y sus Efectos en la Población Trabajadora y sus Protocolos Específicos y se dictan otras disposiciones. Diario Oficial, Bogotá.

Comunidad Andina de Naciones. (2004). Decisión 584. Instrumento Andino de Seguridad y Salud en el Trabajo. Guayaquil: Comunidad Andina de Naciones. 
Espejo, L., Perico, A., \& Quesada, M. (2014). Educando para el bienestar docente: sistematización de experiencias de Terapia Ocupacional en el estudio de los factores de riesgo psicosocial que afectan a los y las docentes de dos colegios distritales de la localidad de Engativá en la ciudad de Bogotá (Monografia). Universidad Nacional de Colombia, Bogotá.

Gil, J. A. M., Isidoro, S. G., \& Sánchez, V. O. C. (2016). Análisis del riesgo laboral psicosocial en una empresa metalmecánica de Colombia, una experiencia de intervención apoyada de modelos basados en la evidencia. TOG, 13(23), 1-21.

Gil-Monte, P. R. (2012). Riesgos psicosociales en el trabajo y salud ocupacional. Revista Peruana de Medicina Experimental y Salud Pública, 29(2), 237-241.

Gutiérrez-Bolaño, E. N., Bermúdez-Lugo, M., Moreno-Chaparro, J., \& Guzmán-Suárez, O. B. (2019). Estrategias de transformación en organizaciones saludables. Revista Brasileira de Medicina do Trabalho, 17(3), 394-401.

Helfrich, C. (2016) Principios del aprendizaje y del cambio de conducta. In B. Shell, G. Gillen \& M. Sacffa (Eds.), Willard \& Spackman Terapia Ocupacional (pp. 588-603). Georgia: Panamericana.

Icontec International. (2010). Guidance for hazard identification and occupational health and safety risk assessment. Bogotá: Icontec.

Kalimo, R., El-Batawi, M., \& Cooper, C. (1987). Psychosocial factors at work. Geneva: Publicaçóes.

Kielhofner, G. (2002). A model of human occupation: theory and application. Illinois: Lippincott Williams $\&$ Wilkins.

Kielhofner, G. (2004). Terapia ocupacional: modelo de ocupación humana: teoría y aplicación. Illinois: Médica Panamericana.

King, P., \& Olson, D. (2016). Trabajo. In B. Shell, G. Gillen \& M. Sacffa. (Eds.), Willard \& Spackman Terapia Ocupacional (pp. 678-696). Georgia: Panamericana.

Law, M., Cooper, B., Strong, S., Stewart, D., Rigby, P., \& Letts, L. (1996). The person-environmentoccupation model: A transactive approach to occupational performance. Canadian Journal of Occupational Therapy, 63(1), 9-23.

Leka, S., \& Jain, A. (2010). Health impact of psychosocial hazards at work: an overview. Nottingham: World Health Organization.

López, B. P. (2004). Terapia ocupacional en discapacitados físicos: teoría y práctica. Espanha: Editorial Médica Panamericana.

Marmot, M., Allen, J., Bell, R., Bloomer, E., Goldblatt, P., \& Consortium for the European Review of Social Determinants of Health and the Health Divide. (2012). WHO European review of social determinants of health and the health divide. Lancet, 380(9846), 1011-1029.

Molina, P. D., \& Arnaiz, B. N. (1998). Terapia ocupacional en salud mental: principios y práctica. Barcelona: Masson.

Moreno, B., \& Báez, C. (2010). Factores y Riesgos Psicosociales, formas, consecuencias, medidas y buenas prácticas. Madrid: Universidad Autónoma de Madrid.

Moreno Jiménez, B. (2011). Factores y riesgos laborales psicosociales: conceptualización, historia y cambios actuales. Medicina y Seguridad del Trabajo, 57(Suppl.1), 4-19.

Munar, P. L. (2017). Efectividad de la intervención de Terapia Ocupacional en la reinserción laboral de pacientes con trastorno de estrés postraumático (TEPT) de origen laboral. Revista Ocupación Humana, 13(1), 54-63.

O’Connor, T., Flynn, M., Weinstock, D., \& Zanoni, J. (2014). Occupational safety and health education and training for underserved populations. New Solutions, 24(1), 83-106.

Organización Internacional del Trabajo - OIT. (1985). C161 - Convenio sobre los servicios de salud en el trabajo. In Anais do 70 a Conferencia General de la Organización Internacional del Trabajo. Ginebra: OIT.

Organización Internacional del Trabajo - OIT. (2019). Trabajo decente. Recuperado el 5 de marzo de 2013, de http://www.oit.org/global/topics/decent-work/lang--es/index.htm

Organización Mundial de La Salud - OMS. (1986). Carta de Ottawa: Primeira Conferência Internacional Sobre Promoção da Saúde. Ottawa: Organización Mundial de La Salud. 
Organización Mundial de La Salud - OMS. (2010). Entornos Laborales Saludables : Fundamentos y Modelo de la OMS. Ginebra: Organización Mundial de La Salud. p. 144.

Organización Mundial de La Salud - OMS. (2019). Salud Ambiental y Entornos Saludables. Recuperado el 5 de marzo de 2013, de https://www.paho.org/col/index.php?option=com_content\&view=category\&layout=blog\&layout=+ blog\&id=388

Paganizzi, L. (2007). Terapia ocupacional psicosocial: escenarios clínicos y comunitarios. Buenos Aires: Polemos.

Paterson, C. F. (2008). A short history of occupational therapy in psychiatry. In J. Creek \& L. Lougher (Eds.), Occupational Therapy and Mental Healt (pp. 3-16). Edimburgh: Elsevier/Churchill Livingston.

Peñas Felizzola, O. L. (2005). La investigación en terapia ocupacional: historia, actualidad y perspectivas. Colombia: Universidad Nacional de Colombia.

Polonio, B., Durante, P., \& Noya, B. (2001). Conceptos fundamentales de terapia ocupacional. Madrid: Médica Panamericana.

Primeau, L. (2016). Juego y ocio. In B. Shell, G. Gillen \& M. Sacffa. (Eds.), Willard \& Spackman Terapia Ocupacional (pp. 702-703). Georgia: Panamericana.

Reason, J. T. (1997). Managing the risks of organizational accidents. Inglaterra: Ashgate.

Reitz, M. (2016). Teorías de promoción de la salud. In B. Shell, G. Gillen \& M. Sacffa (Eds.), Willard \& Spackman Terapia Ocupacional (pp. 574-587). Georgia: Panamericana.

Rincón Herrera, E., Ramos Santos, R., \& Sánchez-Ortiz, M. M. (2007). Terapia ocupacional intervención en distintas patologías. Espanha: Formación Alcalá.

Romero Ayuso, D. M. (2010). El sentido de la ocupación. TOG, 7(Supl. 6), 59-77.

Scaffa, M. (2016a). Proceso grupal e intervención grupal. In B. Shell, G. Gillen \& M. Sacffa. (Eds.), Willard \& Spackman Terapia Ocupacional (pp. 437-451). Georgia: Panamericana.

Scaffa, M. (2016b). Regulación emocional. In B. Shell, G. Gillen \& M. Sacffa. (Eds.), Willard \& Spackman Terapia Ocupacional (pp. 874-881). Georgia: Panamericana.

Shell, B., Gillen, G., \& Sacffa, M. (2016). Willard \& Spackman Terapia Ocupacional. Georgia: Panamericana.

Solet, J. (2016). Sueño y descanso. In B. Shell, G. Gillen \& M. Sacffa. (Eds.), Willard \& Spackman Terapia Ocupacional (pp. 528-533). Georgia: Panamericana.

Trujillo, A. (2002). Terapia ocupacional: conocimiento y práctica en Colombia. Bogotá: Universidad Nacional de Colombia.

Vaquero, M. (2011). Terapia Ocupacional en centros de rehabilitación psicosocial: a propósito de un caso clínico de esquizofrenia paranoide. TOG, 8(14), 1-12.

Vidal Sánchez, M. I. (2015). Bibliographic review on burnout and occupational therapy: vulnerability facing opportunity. TOG, 12(21), 1-29.

\section{Contribución de los Autores}

Karen Danniela Medina Murillo aportó en concepción del texto, análisis, redacción. Olga Beatriz Guzmán Suárez aportó en la concepción del texto, análisis y revisión. Jaime Moreno-Chaparro en concepción del texto, análisis, redacción y organización de fuentes.

Todos los autores aprueban la versión final del texto.

\section{Autor para la correspondencia}

Karen Danniela Medina Murillo

e-mail: kdmedinam@unal.edu.co 